\title{
Performance Analysis of Cloud Computing Platform
}

\author{
Swapna Addamani \\ Dept of Computer Science \& Engg, R\&D \\ East Point College of Engineering \& Technology, \\ Bangalore, India.
}

\author{
Anirban Basu, PhD. \\ Dept of Computer Science \& Engg, R\&D \\ East Point College of Engineering \& Technology, \\ Bangalore, India.
}

\begin{abstract}
This paper discusses the different approaches to performance analysis of cloud computing platforms and proposes a queuing model for performance analysis of a web application. In this model, the cloud computing platform is modeled as multiple queues and the virtual machines VMs are modeled as service centers. In this model, the instances act as virtual machines and VMs run on servers, its number decided a priori before running an application. The performance of closed jobs executing on cloud computing platform was analyzed for different distributions. The model is based on the Reserved Instances behavior of applications on Amazon EC2.
\end{abstract}

\section{Keywords}

Cloud Computing, Performance Evaluation, Benchmarking, Closed Queuing Network, JMT Tool.

\section{INTRODUCTION}

Cloud computing provides utility oriented IT services on "pay-per-use" basis [1]. "The Cloud" has a large pool of resources and provides a development platform which can be reconfigured dynamically to adjust to a variable load for better performance and for optimum utilization. Users submit their requests for computing resources such as CPU, RAM, disk, application, infrastructure software, etc. which are provisioned in the cloud platform without the users being aware about the details of execution environment. The important entities involved in Cloud computing are [1]: Cloud Server (CS) or Cloud Service Provider (CSP): Is a pool of resources in a data centre and provides huge processing power and variety of services to the Clients. Clients who demands services from CS/CSP.The services may include computing power, storage resources, applications, and processes. Cloud based services have three service models which are:

- Infrastructure-as-a-Service (IaaS),

- $\quad$ Platform-as-a-Service (PaaS) and

- $\quad$ Software-as-a-Service (SaaS).

Examples of IaaS cloud include Amazon EC2, IBM Cloud. Microsoft Azure and Google AppEngine are examples of PaaS cloud. Examples of SaaS include Gmail, Google Docs.

An important characteristic of a cloud-based service is virtualization of physical resources which enables the execution of multiple jobs on the same, shared physical computing resource. This makes cloud based service economical to both cloud service providers and cloud users. Cloud service providers can reduce their costs of service delivery by resource consolidation (through virtualization) while users of cloud service reduce the cost of computing due to a pay-as-you-go pricing model, where the users are charged based on their computing demand and duration of resource usage.
With the availability of several options and deployment models, understanding and analyzing the performance have become extremely critical in cloud computing. Commercial success of any Cloud Computing platform depends upon its ability to deliver guaranteed Quality of Services (QoS) and evaluating the performance by measurements, simulation or by modeling is becoming important. In this paper, we present an approach for studying performance of IaaS cloud computing platform and propose a queuing model for performance evaluation with closed (web applications) class of jobs.

\section{EARLIER WORK}

Measurement is used for selecting the hardware and software configuration of any computer system prior to procurement and done by running Benchmark programs. With the usage of cloud computing picking up, more and more users are seeking answers to questions on which type of cloud service they should choose and from which vendor. While work is going on designing appropriate benchmarks, so far Phoronix Test Suite [2] has gained prominence. Performance evaluation of Cloud Computing platforms by measurement using Benchmark programs has been conducted. Jorg Schad, Jens Dittrich, Jorge-Arnulfo Quian'e-Ruiz carried out a study of the performance variance of the most widely used Cloud infrastructure (Amazon EC2) from different perspectives [3]. Micro benchmark programs were used to measure the performance variance in CPU,I/O and Network. In [4] authors focus on performance measurement and analysis of network I/O applications (network-intensive applications) in a virtualized cloud. To maximize the benefit and effectiveness of server consolidation and application consolidation, the authors show that performance improvement can be high by strategically collocating network I/O applications. Results of performance analysis of Cloud Computing Services for ManyTasks Scientific Computing on four commercial Cloud Computing platforms have been reported in [5] and performance measurement of scientific applications on Amazon EC2 has been carried out in [6]. In [7], Huber et. al. Present experimental results on two popular state-of-the-art virtualization platforms, Citrix Xen Server 5.5 and VMware ESX 4.0, as representatives of the two major hypervisor architectures. Based on these results, they propose a basic, generic performance prediction model for the two different types of hypervisor architectures.

Performance Evaluation of Amazon EC2 focusing on non functional properties has been reported in [8]. Simulation has also been one of the evaluation techniques for performance evaluation of Cloud Computing platforms. CloudSim[9] is one of the most popular simulation tool. Performance Evaluation on Open Cirrus Cloud Computing test bed located at the University of Illinois using tools to mimic distributed 
users and cloud facilities was carried out in 2009 as reported in [10] Application of CloudSim to study Cloud Computing environments has been reported in [11]. Modeling of a Cloud Computing platform for the performance analysis has been done by few researchers. In [12] Brebner and Liu have used a Service Oriented Performance Modeling Technology for modeling the performance and scalability of Service Oriented Applications architected for a variety of platforms. In [13] Hamzeh et. al. discuss use of $\mathrm{M} / \mathrm{G} / \mathrm{m}$ queues for studying performance and derive analytical solutions. In [14], the authors describe a Markov chain based approach for the performance and availability analysis of cloud provided services for IaaS deployment model. In [15], Chen and Li propose a queuing-based model for performance management on cloud and carried out measurements on an experimental set up. Analysis of a cloud system using Queuing Theory and $\square \square-$ calculus for improving the cluster utilization keeping security aspects in mind has been discussed in [16]. Kaiqi Xiong and Harry Perros in [17] have proposed a Queuing Network model for studying the performance of a Cloud Computer especially the response time. In this paper, we present an approach for studying performance of IaaS cloud computing platform and propose a queuing model for performance management with closed (for web applications) class of jobs. The model has been analysed using JMT tool [18].

\section{QUEUING MODEL FOR THE CLOUD}

An application runs on the virtual machines on cloud using Virtualization software such as Xen, KVM etc. [4]. It is through virtual machines (VM) that the applications share computing resource on a cloud. As shown in Figure 1, when an user sends a request to a web application on cloud, the request is sent to the Cloud Controller. The dispatcher in Cloud Controller forwards the request to the queue of the target application. The instances of the target application run on VMs which act as servers to process the requests in the queue.

The system is modeled as a queue and the VMs as servers. The number of instances needed to be used in the form of
VMs is decided a priori by the Web Application Developer. The user requests coming from an application join the queue and await service. A Cloud Computing platform can be modeled as consisting of multiple queues for different applications and the Web Application Provider uses the Reserved Instances feature on Amazon EC2 [12] to specify the number of VMs for each application to deliver the QOS as per the SLA with the users. The user requests can be served in many possible orders, such as first come first served, last come first served, shortest processing time first, random order, round robin, and so on. However, the first come first served is still the predominant order.

When an application is deployed on a cloud, the Cloud Controller forms a queue for it to hold the client requests. VMs are created by Cloud Controller on Cloud Node(s) as decided by the Web Application Provider. The number of VMs created can be specified by service level agreement or an empirical value is used in case nothing is specified in service level agreement. All the VMs of an application run on either a single Cloud Node or multiple Cloud Nodes, and each of them has the same computing resource. Regardless of the physical location of VMs, there is an instance of the web application running on each of them.

For example, in Figure 1, application A has $n$ instances each of which is running on a VM. Similar to A, application B has $\underline{m}$ instances. All the VMs of A and $\mathrm{B}$ can run on a single Cloud Node or run on multiple Cloud Nodes, and they compose two clusters. Queue A and Queue B on Cloud Controller respectively are associated with applications A and B. The Closed Queuing Model used to analyse the behaviour is shown in Figure 2.

\section{APPLICATION BENCHMARKS}

Two web applications: Tourism Management System and Product Search Engine were run to measure parameters pertaining to the Queuing Model viz, The Think Time and Service Time. These were measured by using software monitors at the appropriate places in the two application

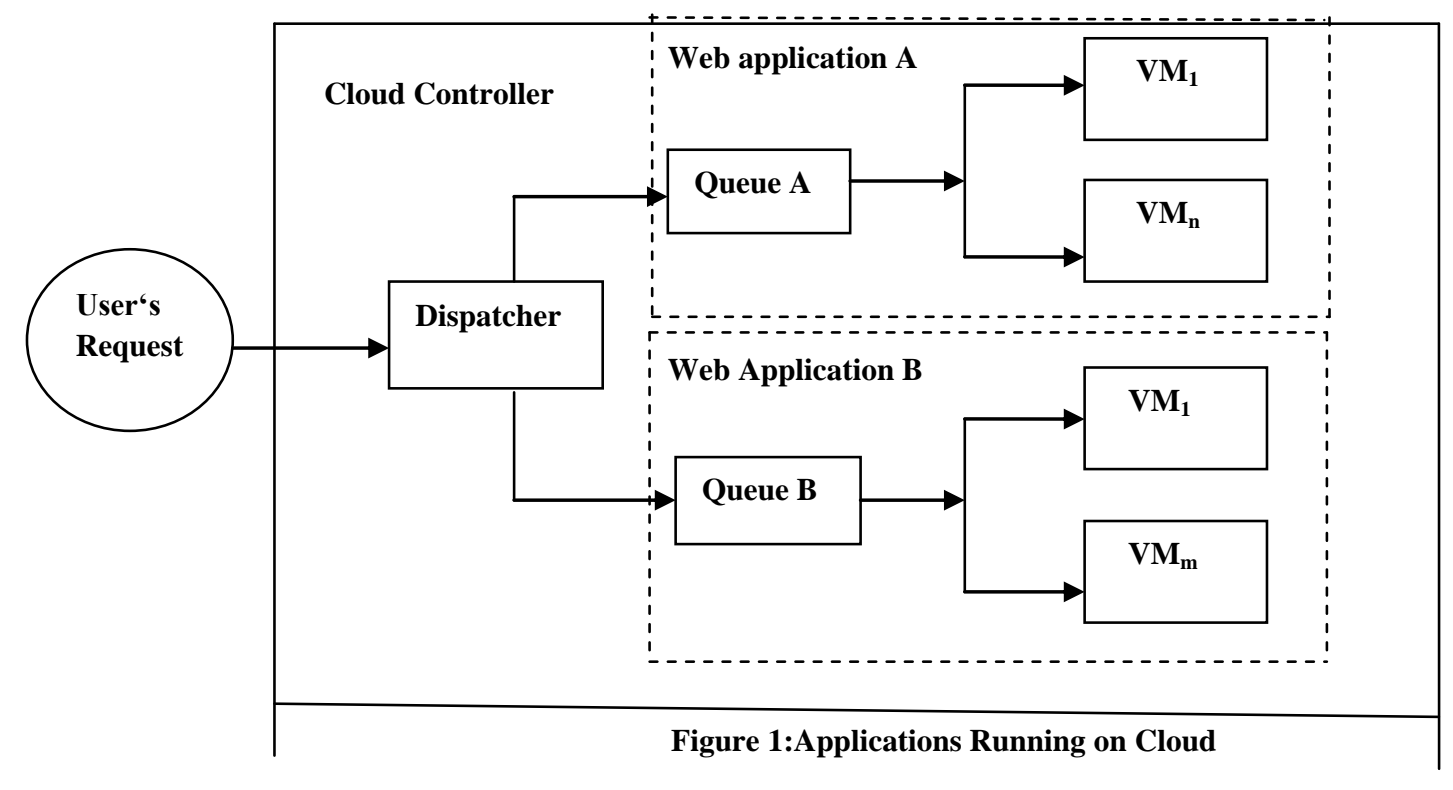




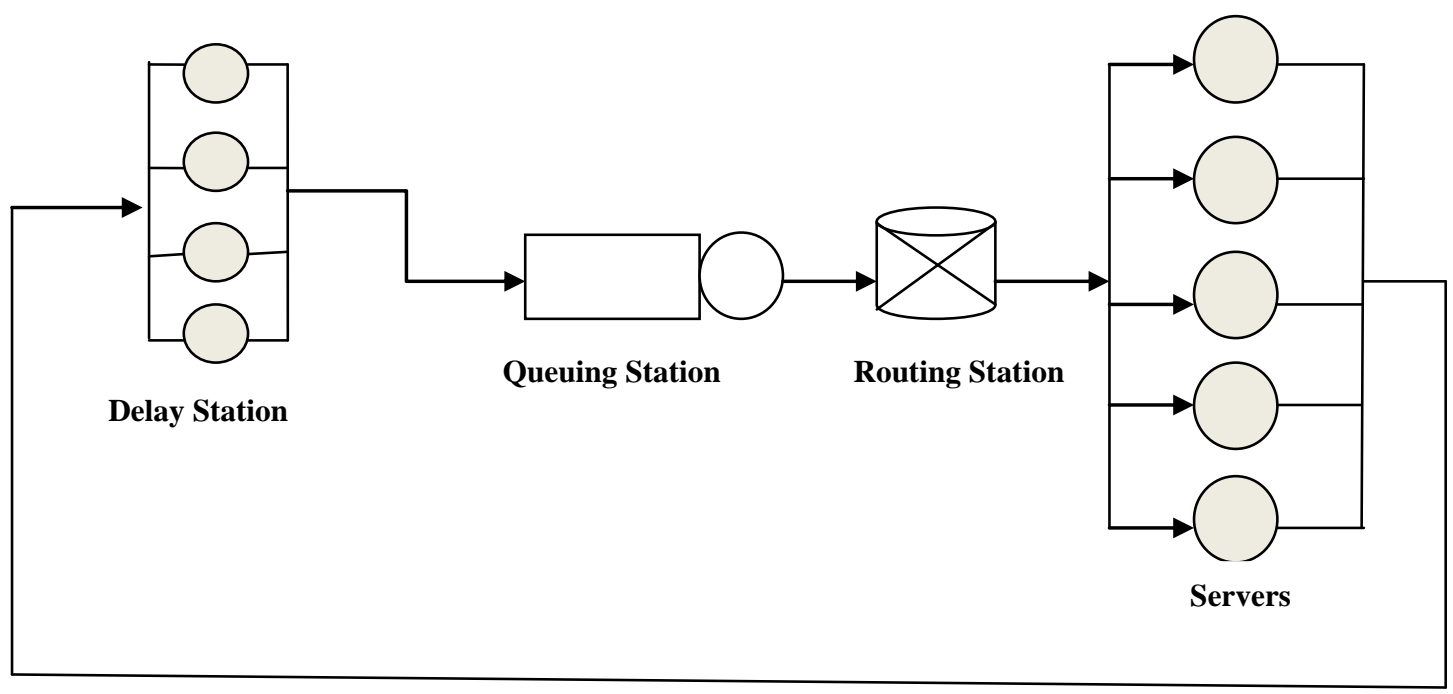

Figure 2: Closed Queuing Model

\subsection{Tourism Management System}

It automates the operations of Tourism Administration, which enables the travel industry to sell tours efficiently, provide a consistent high level of customer service and simple back office administration. This system can be used as an application for the tourism with an extensive range of standard features for real-time reservation software, booking systems, web publication and efficient back-office administration for tour operators, incoming agents, destination management companies and other tourism management department of state government. The vendors who register for providing a service can be given a feature to add a page to publish their details.

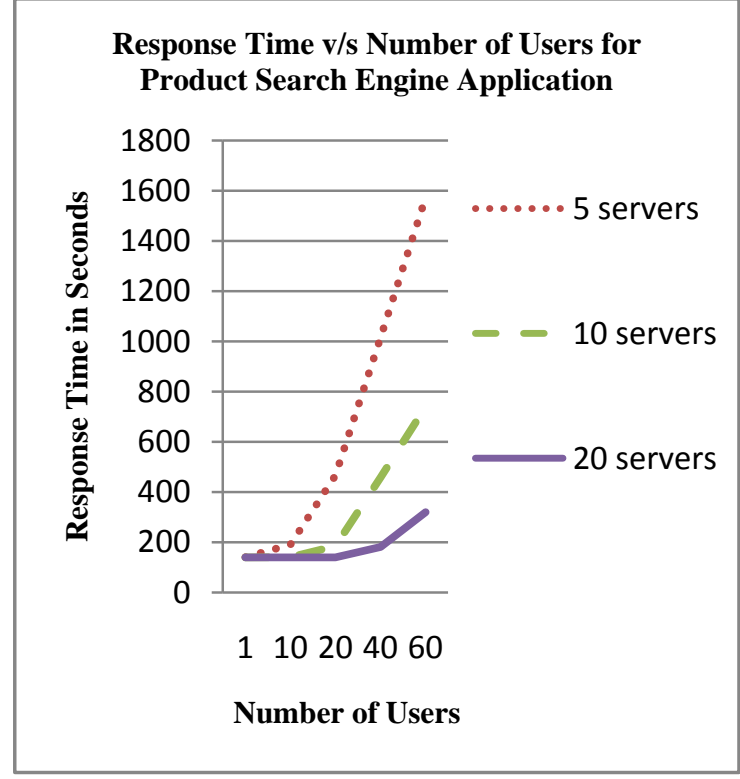

Figure 3: Product Search Engine

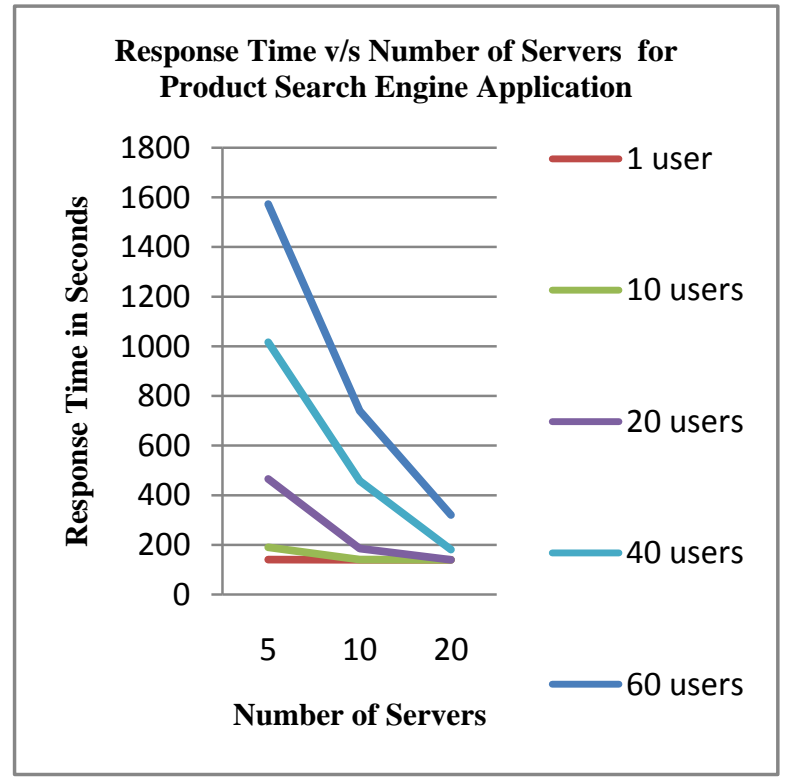

Figure 4:Product Search Engine 


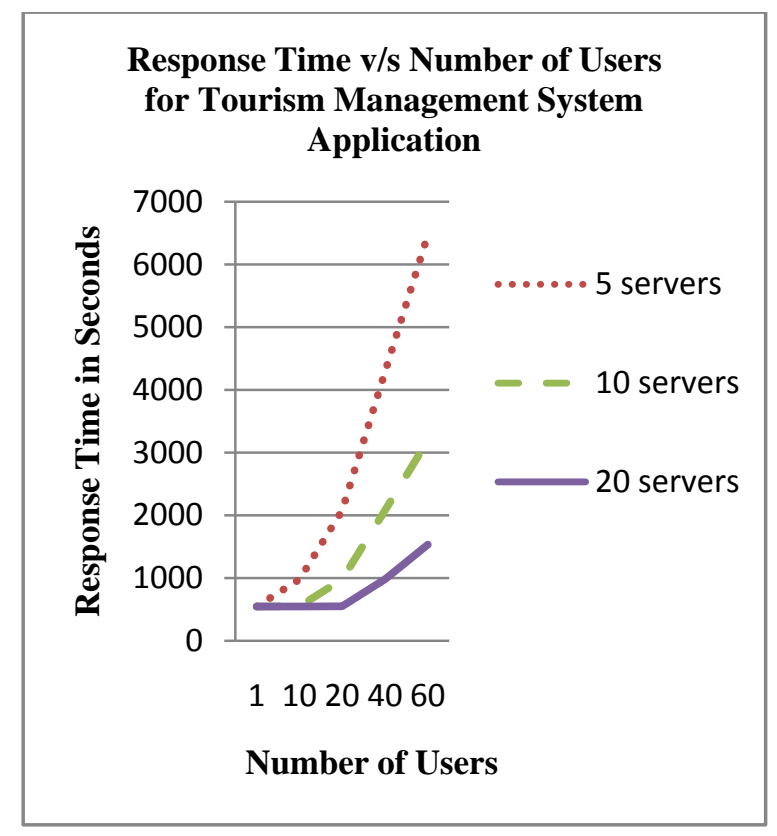

Figure 5:Tourism Management System

\subsection{Product Search Engine}

In product based search engine, user allows searching the different products and if he wishes to purchase he can proceed. Here the product details are stored in the database of that application. Once user is registered to this web page, he can search the products based on interest. This application gives separate login page for each user.

\subsection{Measurement of parameters}

The web applications were run and data collected on parameters which were intrinsic characteristic of the applications and independent of the environment of their execution. The parameters of interest were Think Time and Service Time and these were measured by using software instrumentation techniques, The values collected on the two applications were analyzed for different operations and their characteristics determined by analysis using MINITAB software. The result of the data analysis shows that the Think Time for both the applications follows Weibull Distribution with different values of shape and scale parameter whereas the Service Time follows Exponential Distribution with different values of scale parameter.

\section{PERFORMANCE ANALYSIS USING QUEUING MODEL}

The closed queuing model was analyzed using JMT with different distributions of Think Time and Service Time using multi server queuing model shown in Figure 2 using FCFS routing discipline. The number of servers and the number of users were varied and the model analyzed for different values. The results for the application are shown in Figures 3 and 4 which shows the variation of Response Time with the number of users and number of servers for the with Product Search Engine. The performance of the Tourism Management System is shown in Figures 5 and 6

\section{CONCLUSION}

The paper presents a closed Queuing Model for performance analysis of Cloud Computing Platforms. Software monitoring
Response Time v/s Number of Servers for Tourism Management System Application

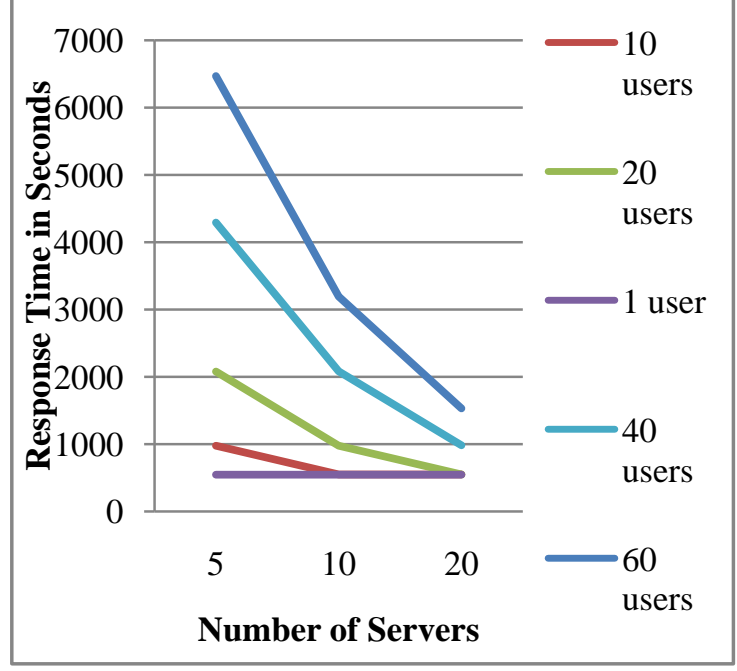

Figure 6: Tourism Management System

was used to measure model parameters on the two web applications and the model was analyzed using JMT tool. The variation of the response time with the number of users and with the number of instances (or Virtual Machines) were studied and the results can be used to determine the optimal number of users and the number of VMs for a desirable value of the response time.

\section{REFERENCES}

[1] Rajkumar Buyya and Karthik Sukumar, Platforms for Building and Deploying applications for Cloud Computing, CSI Communications, May 2011, Vol. 35. No. 1.pp. 6-11

[2]www.phoronix-test-suite.com

[3] Jorg Schad, Jens Dittrich, Jorge-Arnulfo Quian`e-Ruiz, "Runtime Measurements in the Cloud: Observing, Analyzing, and Reducing Variance", Proceedings of VLDB Endowment. Vol.3. No. 1.pp. 460- 471

[4]Y Mei. L Liu, X Pu, S Sivathanu. "Performance Measurements and Analysis of Network I/o Applications in Virtualized Cloud". Proc. IEEE International Conference on Cloud Computing, Miami, Fl, July 2010

[5] Alexandru Iosup, Simon Ostermann, M. Nezih Yigitbasi, Radu Prodan, Thomas Fahringer, and Dick H.J. Epema, "Performance Analysis of Cloud Computing Services for Many-Tasks Scientific Computing", IEEE Trans. On Parallel and Distributed Systems, Vol. 22, No.6. June 2011, pp. 931-945.

[6] Keith R. Jackson, Lavanya Ramakrishnan, Krishna Muriki, Shane Canon, Shreyas Cholia, John Shaif, Harvey Wasserman and Nicholas Wright, " Performance Analysis of High Performance Computing Applications on the Amazon Web Services Cloud". Proc. IEEE Second International Conference on Cloud Computing Technologies Science, 2010 
[7] Nikolaus Huber, Marcel von Quast, M Hauck and S Kounev" Evaluating and modeling virtualization performance overhead for cloud environments", 2011

[8] Vladmir Stantchev, "Performance Evaluation of Cloud Computing offerings". Proc. 20092009 Third International Conference on Advanced Engineering Computing and Application in Sciences, pp. 187-192

[9] Rajkumar Buyya, Rajiv Ranjan and Rodrigo N. Calheiros1,"Modeling and Simulation of Scalable Cloud Computing Environments and the CloudSim Toolkit: Challenges and Opportunities", Proc. of International Conference on High Performance Computing \& Simulation, 2009, June 2009. pp. 1-11

[10]. Ahmed Khurshid, Abdullah Al-Nayeem, and Indranil Gupta," Performance Evaluation of the Illinois Cloud Computing Testbed", Technical Report, Department of Computer Science University of Illinois at UrbanaChampaign, 2009

[11] Rodrigo N. Calheiros, Rajiv Ranjan, and Rajkumar Buyya," Virtual Machine Provisioning Based on Analytical Performance and QoS in Cloud Computing Environments", Proc. International Conference on Parallel Processing (ICPP), Sept 2011, pp. 295-304

[12] Paul Brebner and Anna Liu,"Performance and Cost Assessment of Cloud Services", Proc. of Int. Conference on Service Oriented Computing, 2010, pp. 39-50
[13] Hamzeh Khazaei,. Jelena Misic and Vojislav B Misic, "Modelling of Cloud Computing Centers Using M/G/m Queues", Proc. $201131^{\text {st }}$ International Conference on Distributed Computing Workshops, pp.87-92

[14] K S Trivedi, Rahul Ghosh and Vijay K Naik, "Performance and Availability Analysis for Infrastructure as a Service Cloud", Proc. CONSEG 2011, International Conference on Software Engineering, Bangalore, Feb 2011, pp. 106-111

[15] Hao-peng CHEN, Shao-chong LI," A Queuing-based Model for Performance Management on Cloud", Proc. 2010 6th International Conference on Advanced Information Management and Service (IMS), pp. 83-88

[16] A M Alijohani, D R W Holton, I Awan and J S Alanazi, "Performance Evaluation of Local and Cloud Deployment of Web Clusters". Proc. 2011 International Conference on Network-Based Information Systems, pp.274- 278

[17] Kaiqi Xiong and Harry Perros, "Service Performance and Analysis in Cloud Computing", Proc. of the IEEE Congress on Services-IEEE Cloud 2009.

[18] Marco Bertoli, Giuliano Casale, Giuseppe S, JMT: performance engineering tools for system modeling, ACM SIGMETRICS Performance Evaluation Review, Volume 36 Issue 4, March 2009, pp. 10-15 